Running head: PSYCHOMETRIC PROPERTIES OF SOCIAL ANXIETY MEASURES IN ASD

Measuring Social Anxiety in Adults with Autism Spectrum Disorder: Psychometric Properties of Self-Report Instruments

Authors: Kelsie A. Boulton ${ }^{1,2}$, \& Adam J. Guastella ${ }^{1,2}$

1. Autism Clinic for Translational Research, Brain and Mind Centre, Children's Hospital Westmead Clinical School, Faculty of Medicine and Health, University of Sydney, Camperdown, New South Wales, 2050, Australia.

2. Child Neurodevelopment and Mental Health Team, Brain and Mind Centre, Children's Hospital Westmead Clinical School, Faculty of Medicine and Health, University of Sydney, Camperdown, New South Wales, 2050, Australia.

Please address correspondence to:

Prof Adam Guastella

Brain \& Mind Centre

The University of Sydney

100 Mallett Street

Camperdown, NSW 2050

Australia

adam.guastella@sydney.edu.au 


\begin{abstract}
Adults with autism spectrum disorder (ASD) are at elevated risk for social anxiety disorder (SAD). Limited information exists on the appropriateness of using social anxiety instruments with these adults. This study examines psychometric properties of self-report social anxiety instruments in autistic adults without intellectual disability, compared to adults with SAD. Additionally, we compared instrument scores between autistic adults with a dual diagnosis of SAD and adults with SAD only. Adults diagnosed with $\operatorname{SAD}(N=316)$ or ASD $(N=102)$ were recruited from the Brain and Mind Centre in New South Wales, Australia. Sixty autistic participants received a diagnosis of SAD. Participants completed the Liebowitz Social Anxiety Scale-self-report, the Social Interaction Anxiety Scale, the Social Phobia Scale, and the Brief Fear of Negative Evaluation Scale. All instruments showed excellent internal consistency in autistic adults. Summary scores across instruments were strongly correlated within both groups, and the strength of these correlations were equivalent between ASD and SAD. For all instruments, the performance of autistic adults with a dual diagnosis of SAD was comparable to adults diagnosed with SAD only. Findings suggest that these instruments display adequate psychometric properties in autistic adults without intellectual disability, supporting the use of these instruments in clinical services.
\end{abstract}




\section{Lay Abstract}

Research shows that autistic adults are likely to experience social anxiety, and are at increased risk of being diagnosed with social anxiety disorder. However, we have little information about whether self-report instruments measuring social anxiety (e.g., questionnaires and rating scales) can be appropriately used with autistic adults. We examined the appropriateness and reliability of four commonly used self-report social anxiety instruments in a large group of autistic adults without intellectual disability, compared to a large group of adults with social anxiety disorder. We also compared scores on these instruments between autistic adults who were also diagnosed with social anxiety disorder and adults who were diagnosed with only social anxiety disorder. We found similar patterns of results between autistic adults and adults with social anxiety disorder, suggesting that these self-report instruments can be used to measure social anxiety in autistic adults. We also found that the autistic adults who were diagnosed with social anxiety disorder showed similar scores on all instruments to the adults who were diagnosed with only social anxiety disorder. These findings have implications for clinical services, as they show that these instruments are appropriate to use with autistic adults without intellectual disability. Given that many autistic adults experience social anxiety, clinical services may benefit from using these instruments to identify and assess social anxiety in autistic adults.

Keywords: Autism Spectrum Disorder; Social Anxiety Disorder; Adults; Social anxiety; Reliability; Rating scales 


\section{Introduction}

There is a growing awareness of the mental health concerns that occur alongside autism spectrum disorder (ASD). Approximately $70 \%$ of young autistic adults meet diagnostic criteria for at least one Axis I disorder (Lugnegard, Hallerback, \& Gillberg, 2011; Vasa \& Mazurek, 2015), such as depression and anxiety. These mental health issues are a major contributor to disability and distress in adults with ASD (Howlin, Goode, Hutton, \& Rutter, 2004; Howlin \& Moss, 2012; Park et al., under review), compromising educational, social and occupational functioning (Howlin, Moss, Savage, \& Rutter, 2013; Park et al., 2019; Shattuck et al., 2012). Given the social communication and interaction difficulties central to ASD, it is unsurprising that many autistic individuals experience significant anxiety about social situations. Social anxiety disorder (SAD) is common in ASD, with comorbidity estimates of up to $80 \%$ reported (Hollocks, Lerh, Magiati, Meiser-Stedman, \& Brugha, 2019; Maddox \& White, 2015; Spain et al., 2016). In contrast to the high SAD prevalence rates noted in ASD, SAD rates are estimated at between 7-12\% of the general population (Crome et al., 2015; NICE, 2013). Despite this high prevalence, identification and diagnosis of cooccurring SAD in ASD is often under-recognised, impeding access to treatment (Mazzone, Ruta, \& Reale, 2012).

Autistic adults typically report elevated social anxiety symptoms relative to typically developing adults (Spain, Sin, Linder, McMahon, \& Happé, 2018). (Bejerot, Eriksson, \& Mörtberg, 2014). To illustrate, Maddox and White (2015) found that adults with a primary diagnosis of ASD and without intellectual disability performed similarly to those with SAD on measures of social interaction anxiety, but displayed lower symptom scores on measures tapping into fear of negative evaluation when compared to the SAD diagnostic group. Additionally, relative to adults with a sole diagnosis of ASD, autistic adults who received a 
comorbid diagnosis of SAD displayed significantly higher symptom scores both on measures of social interaction anxiety and fear of negative evaluation.

To date, only one study has investigated the reliability of commonly used self-report social anxiety instruments in autistic adults. In 49 males with ASD and without intellectual disability, Spain et al. (2016) examined the internal consistency of four instruments commonly used to measure social anxiety symptoms, the Liebowitz Social Anxiety Scale-SR (LSAS-SR; Heimberg et al., 1999), the Social Interaction Anxiety Scale (SIAS; Mattick \& Clarke, 1998), the Social Phobia Scale (SPS; Mattick \& Clarke, 1998), and the Brief Fear of Negative Evaluation Scale (BFNE; Leary, 1983). In addition to reporting elevated social anxiety symptoms across instruments, the authors found that all four instruments demonstrated high internal consistency in autistic adults $(\alpha=0.90-0.96)$.

No research to date has comprehensively evaluated the psychometric properties of self-report social anxiety instruments in autistic adults relative to those with SAD, and subsequently compared performance on these measures between diagnostic groups. It has been proposed that self-report measures developed for the general population may not have utility in ASD, given autistic individuals tend to have greater difficulties reporting on their own affective states and emotions (Ainsworth et al., 2020; Volkmar, Lord, Bailey, Schultz, \& Klin, 2004). As such, an important first step is to ascertain whether self-report social anxiety instruments operate similarly for those with ASD, in comparison to those without ASD.

In light of the gaps in the current literature, this study aimed to examine the psychometric properties of multiple self-report social anxiety instruments commonly used in clinical practice and research in an ASD cohort without intellectual disability, compared to a SAD cohort. Given their robust psychometric properties within the SAD literature, and their increasing use within the ASD literature, the self-report instruments selected for inclusion 
were the LSAS-SR, the SIAS, the SPS and the BFNE. Specifically, we evaluated the instruments' internal consistency, item-total correlations and concurrent validity, as well as investigating floor and ceiling effects for each instrument. Additionally, performance on each instrument (indexed by summary scores) was directly compared between autistic adults with a dual diagnosis of SAD and adults with SAD.

We hypothesised that internal consistency would be high for all instruments in both SAD and ASD groups. We further predicted that the summary scores for each instrument would be significantly correlated within each diagnostic group. We also predicted that the measures would show similar summary scores and cut-off values for those diagnosed with ASD and SAD, in comparison to those diagnosed with only SAD.

\section{Method}

\section{Participants}

A cohort of adolescents and adults $(N=418$, Age: $M=24$ years, 3 months, range $15-$ 46 years) were recruited from the Autism Clinic for Translational Research (ACTr), the Anxiety Clinic, and the collocated Headspace clinic at the Brain and Mind Centre, the University of Sydney, between January 2012 and February 2019. Informed written consent was obtained from each participant, and the study was approved by the University of Sydney's Human Research Ethics Committee (2012/1631; 2013/352). Participants presented to the Headspace, anxiety or ACTr clinics for a range of concerns (e.g., social skills development, mental health concerns) and were either self-referred or referred by mental health professionals. Participants met primary diagnostic criteria for SAD $(N=316)$ or ASD $(N=102)$. A total of 60 autistic participants also completed SAD diagnostic assessments and met criteria for SAD. The remaining 42 participants either did not complete the SAD diagnostic assessment $(N=33)$ or did not meet criteria for $\operatorname{SAD}(N=9)$. All participants were 
assessed by research qualified clinicians and diagnoses were made using standardised diagnostic instruments. Participants meeting criteria for SAD completed the Anxiety Diagnostic Interview Schedule (Brown \& Barlow, 2014; Brown, DiNardo, \& Barlow, 1994). Participants diagnosed with ASD met clinical cut-offs on the Autism Diagnostic Observation Schedule-2 $2^{\text {nd }}$ Edition (ADOS-2; Lord, Luyster, Gotham, \& Guthrie, 2012) and a clinical interview assessing DSM-5 criteria. The Wechsler Test of Adult Reading (Wechsler, 2001) was used as an estimate of pre-morbid intellectual quotient (IQ) for screening the presence of intellectual disability in participants. All participants were screened and excluded from the study if they had an intellectual disability (IQ<70), a neurological condition, or current substance dependence. Specific data on socioeconomic characteristics and ethnicity were not collected for the full sample.

\section{Measures}

\section{The Liebowitz Social Anxiety Scale - Self-Report (LSAS-SR; Heimberg et al.,} 1999)

The LSAS-SR is a 24-item questionnaire designed to measure anxiety and avoidance of social situations and social interactions. While originally designed as a clinicianadministered measure relying on the judgment of trained raters, the self-report version of the LSAS is psychometrically sound and commonly used within the SAD literature (Baker, Heinrichs, Kim, \& Hofmann, 2002; Gomez \& Watson, 2017). Respondents are asked to rate the intensity of fear and anxiety experienced during social situations, as well as how frequently the situation is avoided, using a 4-point Likert scale. Higher scores correspond to higher levels of fear and avoidance, with a total score of 60 or higher indicative of generalised social anxiety (Liebowitz, 1987; Mennin et al., 2002). Two subscale scores, fear and avoidance of social situations, as well as a total score, are derived from the measure. 


\section{The Social Interaction Anxiety Scale and Social Phobia Scale (SIAS/SPS; Mattick \& Clarke, 1998)}

The SIAS and the SPS are self-report questionnaires, developed as companion instruments to measure social interaction anxiety and scrutiny fears. The SIAS consists of 20 items assessing general fears of social interaction and the SPS consists of 20 items assessing fear of being observed by others during daily activities. For both the SIAS and the SPS, respondents are asked to rate how characteristic each item is of them using a 5-point Likert scale. Higher scores correspond to increased social interaction anxiety (SIAS) or increased fear of being scrutinised by other (SPS). Scores of 36 or higher for the SIAS, and 26 or higher for the SPS are indicative of symptom severity in the clinical range (Peters, 2000). For the SIAS, scoring is reversed for three items; all items on the SPS are straightforwardly worded.

\section{The Brief Fear of Negative Evaluation Scale (BFNE; Leary, 1983)}

The BFNE is a 12-item self-report questionnaire measuring the degree to which people experience anxiety at the prospect of being evaluated negatively by others. Respondents indicate how characteristic each item is of them using a 5-point Likert scale. Higher scores correspond to higher levels of fear of negative evaluation. Prior research indicates that the four-reverse scored items show poor psychometric qualities, and recommends using only the eight items with straightforward wording (Carleton, Collimore, McCabe, \& Antony, 2011). As such, we calculated two sets of total scores for the BFNE; BFNE, comprised of all 12 items, and BFNE-S, comprised of the eight straightforwardly worded items. Clinical cut-off scores for the BFNE have not been published; scores of 25 or higher for the BFNE-S are indicative of symptom severity in the clinical range (Carleton et al., 2011).

\section{Statistical Analyses}


All statistical analyses were performed in SPSS, version 26. Missing values for all instruments were imputed using the individual mean of the remaining items in the instrument (Shrive, Stuart, Quan, \& Ghali, 2006). See Supplementary Table 1 for missing data reports. Internal consistency was examined using Cronbach's alpha. A value of 0.7 was considered acceptable, between 0.7 and 0.9 was considered adequate, and a value of $>0.9$ was considered excellent (Nunnally, 1967). Item-total correlations were evaluated using Pearson's productmoment correlation coefficients $(r)$. Items with correlations $<0.3$ were considered poor, items with correlations between 0.3 and 0.5 were considered moderate and items with correlations $>0.5$ were considered strong (Cohen, 1988). The presence of ceiling and floor effects were evaluated based on the percentage of participants in each diagnostic group with the minimum or maximum scores. Floor and ceiling effects were considered present if this percentage was $\geq 15 \%$ (Terwee et al., 2007). The concurrent validity of each instrument was assessed by measuring Pearson correlation coefficients between each instrument. A Pearson correlation coefficient of $>0.8$ was defined as very strong, $0.6-0.79$ as strong, $0.40-0.59$ as moderate, $0.20-0.39$ as weak, and $<0.20$ as very weak (Swinscow, 1996). Fisher $r$-to- $z$ transformations were used to test for differences in the magnitude of the correlation coefficients between the SAD and ASD groups.

Total scores on each instrument were compared between the SAD group and autistic participants who met criteria for SAD (ASD+SAD group) using a one-way between subjects' multivariate analysis of variance (MANOVA). Bonferroni adjustments were applied, and statistical significance was set at $.007(.05 / 7)$ to limit family-wise error. To compare the proportion of participants within the SAD and the ASD+SAD groups who met clinical cutoffs on each instrument, chi-square tests were used. 


\section{Results}

\section{Demographics}

Demographic characteristics are presented in Table 1. Considering first the comparisons between the SAD and total ASD groups, there was a statistically significant difference in sex, $\mathrm{X}_{(1)}^{2}=7.465, p=.006$, such that there was a larger proportion of females in the SAD group. No group differences were present for age, $t(416)=1.776, p=.076$.

Considering the comparisons between the SAD group and the subset of autistic participants with a comorbid SAD diagnosis (ASD+SAD), no group differences were present for sex, $\mathrm{X}^{2}(1)=2.212, p=.137$, or age, $t(374)=1.090, p=.276$.

\section{Table 1}

Demographic characteristics for participants with a diagnosis of social anxiety disorder (SAD), autism spectrum disorder (ASD), and comorbid ASD and SAD.

\begin{tabular}{|c|c|c|c|}
\hline & SAD & ASD & $\mathrm{ASD}+\mathrm{SAD}$ \\
\hline & $N=316$ & $N=102$ & $N=60$ \\
\hline $\operatorname{Sex}(\mathrm{N}, \%$ Female $)$ & $138(43.7 \%)$ & $29(28.4 \%)$ & $20(33.3 \%)$ \\
\hline Age $($ Mean $\pm S D)$ & $24.64 \pm 7.108$ & $23.23 \pm 6.609$ & $23.57 \pm 3.291$ \\
\hline
\end{tabular}

Abbreviations: SAD, social anxiety disorder; ASD, autism spectrum disorder; $S D$, standard deviation.

${ }^{\mathrm{a}, \mathrm{b}} p$-value for Pearson chi-square test (sex) and independent samples $t$-test (age) between groups. 


\section{Ceiling and Floor Effects, Internal Consistency, Item-Total Correlations and Proportion of Scores in Clinical Range}

Table 2 displays ceiling and floor effects, internal consistency and the range of itemtotal correlations for each instrument within the SAD and ASD groups. For both the SAD and ASD groups, no ceiling or floor effects were observed for summary scores on any instrument ( $<15 \%$ of participants displayed a ceiling or floor effect on each instrument). For the SAD group, Cronbach's alpha values $(\alpha)$ obtained for each summary score were excellent, ranging from 0.92 to 0.97 . Similarly, within the ASD group, Cronbach's alpha values $(\alpha)$ obtained for each summary score ranged from 0.90 to 0.96 . These values indicate that the internal consistency for all instruments was excellent in both the SAD and ASD groups.

Considering item-total correlations for each instrument within the SAD group, all individual items exhibited satisfactory (at least moderate) correlations with their respective summary scores, except the correlation between item 5 of the SIAS and the SIAS summary score $(r=0.22)$ and item 4 of the BFNE and the BFNE summary score $(r=0.23)$. A similar pattern was observed within the ASD group, with all individual items displaying satisfactory correlations with their respective summary scores, except the correlations between items 5 and 11 of the SIAS and the SIAS summary score ( $r$ 's $=0.25$ and 0.22 respectively) and item 4 of the BFNE and the BFNE summary score $(r=0.12)$. 


\section{Table 2}

Ceiling and Floor Effects, Internal Consistency and Item-Total Correlations for the LSAS-SR, SIAS, SPS and BFNE in Social Anxiety Disorder (SAD) and Autism Spectrum Disorder (ASD) groups

\begin{tabular}{|c|c|c|c|c|c|c|c|c|}
\hline & \multicolumn{4}{|c|}{ Social Anxiety Disorder $(N=316)$} & \multicolumn{4}{|c|}{ Autism Spectrum Disorder $(N=102)$} \\
\hline & Floor score & Ceiling score & Cronbach's & Item-Total & Floor score & Ceiling score & Cronbach's & Item-Total \\
\hline & $n(\%)$ & $n(\%)$ & $\alpha$ & Correlation Range & $n(\%)$ & $n(\%)$ & $\alpha$ & Correlation Range \\
\hline LSAS-SR Total & $1(0.3)$ & $0(0 \%)$ & 0.97 & $0.40-0.71$ & $0(0 \%)$ & $1(1 \%)$ & 0.96 & $0.34-0.74$ \\
\hline LSAS-SR Fear & $2(0.6 \%)$ & $0(0 \%)$ & 0.94 & $0.43-0.73$ & $0(0 \%)$ & $1(1 \%)$ & 0.94 & $0.50-0.72$ \\
\hline $\begin{array}{l}\text { LSAS-SR } \\
\text { Avoidance }\end{array}$ & $5(1.6 \%)$ & $1(0.3)$ & 0.93 & $0.38-0.69$ & $2(2 \%)$ & $1(1 \%)$ & 0.91 & $0.30-0.68$ \\
\hline SIAS & $0(0 \%)$ & $2(0.6 \%)$ & 0.93 & $0.22-0.82$ & $0(0 \%)$ & $1(1 \%)$ & 0.91 & $0.22-0.80$ \\
\hline SPS & $4(1.3 \%)$ & $1(0.3 \%)$ & 0.94 & $0.51-.073$ & $0(0 \%)$ & $1(1 \%)$ & 0.95 & $0.39-0.83$ \\
\hline BFNE & $1(0.3 \%)$ & $20(6.3 \%)$ & 0.92 & $0.23-0.84$ & $0(0 \%)$ & $2(2 \%)$ & 0.90 & $0.12-0.79$ \\
\hline BFNE-S & $2(0.6 \%)$ & $28(8.9 \%)$ & 0.95 & $0.75-0.85$ & $1(1 \%)$ & $7(6.9 \%)$ & 0.94 & $0.74-0.83$ \\
\hline
\end{tabular}

Abbreviations: LSAS-SR, Liebowitz Social Anxiety Scale, self-report; SIAS, Social Interaction Anxiety Scale; SPS, Social Phobia Scale;

BFNE, Brief Fear of Negative Evaluation Scale 
Table 3 displays summary scores and the proportion of summary scores in the clinical range across instruments within the SAD and ASD groups. Consistent with their clinical diagnosis, the majority of participants within the SAD group met clinical cut-offs on the LSAS-SR, SIAS, SPS and BFNE-S. Similarly, and in line with the elevated social anxiety symptoms reported in ASD, more than half of the participants in the ASD group displayed scores at or above clinical cut-offs on the LSAS-SR, SIAS, SPS and BFNE-S.

\section{Table 3}

Descriptive Statistics and Proportion of Scores in Clinical Range across instruments in Social Anxiety Disorder (SAD) and Autism Spectrum Disorder (ASD) groups

\begin{tabular}{|c|c|c|c|c|}
\hline & \multicolumn{2}{|c|}{$\begin{array}{l}\text { Social Anxiety Disorder } \\
\qquad(N=316)\end{array}$} & \multicolumn{2}{|c|}{$\begin{array}{l}\text { Autism Spectrum Disorder } \\
\qquad(N=102)\end{array}$} \\
\hline & $\mathrm{M}(S D)$ & $\begin{array}{c}\text { Proportion meeting } \\
\text { clinical cut-offs } n(\%)\end{array}$ & $\mathrm{M}(S D)$ & $\begin{array}{c}\text { Proportion meeting } \\
\text { clinical cut-offs } n(\%)\end{array}$ \\
\hline LSAS-SR & 75.59 & & 71.15 & \\
\hline Total & (30.33) & $227(71.8 \%)$ & (29.39) & $67(65.7 \%)$ \\
\hline LSAS-SR & 40.07 & & 37.47 & \\
\hline Fear & (15.55) & -* & (16.04) & -* \\
\hline LSAS-SR & 35.53 & & 33.69 & \\
\hline Avoidance & (15.72) & -* & (14.61) & _* \\
\hline SIAS & $\begin{array}{l}50.34 \\
(17.11)\end{array}$ & $263(83.2 \%)$ & $\begin{array}{l}46.92 \\
(15.57)\end{array}$ & $73(71.6 \%)$ \\
\hline SPS & $\begin{array}{l}34.27 \\
(18.35)\end{array}$ & $210(66.5 \%)$ & $\begin{array}{l}30.64 \\
(19.04)\end{array}$ & $57(55.9 \%)$ \\
\hline
\end{tabular}




$\begin{array}{lcccc} & 47.98 & -* & 43.41 & -* \\ & (9.99) & & (10.06) & \\ \text { BFNE } & 30.59 & 256(81 \%) & 27.56 & \\ \text { BFNE-S } & (8.26) & & (8.59) & \\ & & & & \\ \end{array}$

Note: *Indicates no cut-off scores are available. Abbreviations: LSAS-SR, Liebowitz Social Anxiety Scale, self-report; SIAS, Social Interaction Anxiety Scale; SPS, Social Phobia Scale; BFNE, Brief Fear of Negative Evaluation Scale

\section{Concurrent Validity}

The correlations among each of the instruments for the SAD and ASD groups are displayed in Supplementary Tables 2 and 3. Concurrent validity was demonstrated by moderate to strong positive correlations between all instruments in both the SAD and ASD groups. All correlations were highly significant in both groups $(p<.001)$. Fisher's $r$-to- $z$ transformation further revealed the magnitude of these correlations by comparing each pairwise correlation between the SAD and ASD groups. There were no statistically significant differences between the SAD and ASD group for any of the correlation coefficients, indicating that the correlations were of a similar magnitude across clinical groups. See Supplementary Table 4 for $z$-scores.

\section{Differences in Instrument Summary Scores and Proportion of Participants Meeting Clinical Cut-offs between Diagnostic Groups}

Table 4 presents the mean summary scores and the proportion of scores in the clinical range for the formally diagnosed SAD and ASD+SAD groups. To explore whether summary scores for participants with a primary diagnosis of SAD differed from summary scores for autistic participants with a confirmed diagnosis of SAD, a one-way MANOVA was 
conducted. There were no statistically significant differences between groups on these measures, $F(7,368)=1.67, p=.116$, Wilks' $\Lambda=.97$, partial $\eta 2=.03$. Further, there were no statistically significant differences between groups in the proportion of participants who met clinical cut-offs across instruments ( $p$ values $.054-.583)$. 


\section{Table 4}

Performance across instruments Proportion of Scores in Clinical Range for SAD and ASD+SAD groups

\begin{tabular}{|c|c|c|c|c|c|c|c|c|}
\hline & \multicolumn{4}{|c|}{$\operatorname{SAD}(N=316)$} & \multicolumn{4}{|c|}{$\mathrm{ASD}+\mathrm{SAD}(N=60)$} \\
\hline & M & SD & Range & $\begin{array}{c}\text { Proportion meeting clinical } \\
\text { cut-offs }(\%)\end{array}$ & M & $\mathrm{SD}$ & Range & $\begin{array}{l}\text { Proportion meeting clinical } \\
\text { cut-offs }(\%)\end{array}$ \\
\hline LSAS-SR Total & 75.59 & 30.33 & $0-140$ & $71.8 \%$ & 74.74 & 29.57 & $9-144$ & $68.3 \%$ \\
\hline LSAS-SR Fear & 40.07 & 15.55 & $0-71$ & $-*$ & 39.03 & 15.34 & $9-72$ & -* \\
\hline $\begin{array}{l}\text { LSAS-SR } \\
\text { Avoidance }\end{array}$ & 35.53 & 15.72 & $0-72$ & -* & 35.71 & 14.85 & $0-72$ & -* \\
\hline SIAS & 50.34 & 17.11 & $1-80$ & $83.2 \%$ & 49.20 & 15.22 & $17-80$ & $75 \%$ \\
\hline SPS & 34.27 & 18.35 & $0-80$ & $66.5 \%$ & 31.69 & 19.05 & $2-80$ & $60 \%$ \\
\hline BFNE & 47.98 & 9.99 & $12-60$ & $-*$ & 45.09 & 9.10 & $23-60$ & $-*$ \\
\hline BFNE-S & 30.59 & 8.26 & $8-40$ & $81 \%$ & 29.02 & 30.34 & $12-40$ & $70 \%$ \\
\hline
\end{tabular}

Note: *Indicates no cut-off scores are available. Abbreviations: LSAS-SR, Liebowitz Social Anxiety Scale, self-report; SIAS, Social Interaction Anxiety Scale; SPS, Social Phobia Scale; BFNE, Brief Fear of Negative Evaluation Scale 


\section{Discussion}

This study is the first to evaluate the psychometric properties of the LSAS-SR, SIAS, SPS and BFNE in autistic adults, and to compare performance on these instruments between autistic adults with a confirmed diagnosis of SAD and a clinical population with SAD. Overall, our results suggest that the LSAS-SR, SIAS, SPS and BFNE have adequate psychometric properties in an ASD population, making them useful self-report instruments for assessing social anxiety symptoms in autistic adults without intellectual disability. Moreover, our results indicate that the performance of autistic adults with a confirmed diagnosis of SAD on these instruments is comparable to a clinical population with SAD. Taken together, these findings support the use of these instruments for adult ASD populations in clinical services.

Consistent with our predictions, the psychometric properties of the LSAS-SR, SIAS, SPS and BFNE were sound for autistic adults in the current study. Excellent internal consistency for each instrument was observed in both the ASD and SAD groups. This is consistent with prior psychometric evaluations of these measures in SAD populations (Baker et al., 2002; Carleton et al., 2011; Mattick \& Clarke, 1998). These findings also align with previous findings in adult males with ASD (Spain et al., 2016), providing further support for the utility of these measures in an autistic population. Moreover, there were no ceiling or floor effects for any instrument. When looking at item-level statistics for each instrument within the SAD and ASD groups, the majority of items exhibited satisfactory correlations with their respective summary scores, indicating that the individual items for each instrument can discriminate high-scoring individuals from low-scoring individuals. The items displaying weak item-total correlations were similar for the SAD and ASD groups (items 5 and 11 of the SIAS, item 4 of the BFNE). A possible explanation for these weak item-total correlations may lie in the wording of these items; they are all reverse-scored items. Reverse-scored items 
on both the SIAS and BFNE have been critiqued within the literature, showing weaker relationships with comparison measures and loading on a factor separate from the straightforwardly worded items (Rodebaugh, Woods, \& Heimberg, 2007; Weeks et al., 2005).

Our findings highlight the utility of the LSAS-SR, SIAS, SPS and BFNE as clinical measures in an autistic population. Over $50 \%$ of participants in our autistic cohort exhibited scores in the clinical range across instruments, with over two-thirds meeting clinical cut-offs on the SIAS. These figures are consistent with the elevated prevalence of SAD reported in prior autistic samples (Hollocks et al., 2019; Maddox \& White, 2015; Spain et al., 2016). As predicted, summary scores for all instruments were strongly and significantly correlated in both diagnostic groups, indicating that these instruments are measuring a similar underlying construct of social anxiety in ASD. These findings align with what has been reported in previous SAD (Weeks et al., 2005) and ASD (Spain et al., 2016) cohorts. Moreover, the strength of the correlations among each of the instruments were similar between the SAD and ASD groups, further indicating that these measures demonstrate adequate concurrent validity in an autistic population. Taken together, these findings support the use of self-report instruments for assessing social anxiety symptoms in autistic adults without intellectual disability.

In line with our predictions, summary scores for each instrument did not differ when we compared participants with a clinical diagnosis of SAD to autistic participants who also had a confirmed diagnosis of SAD. Further, when looking at the proportion of participants scoring in the clinical range for each instrument, there were no differences as a function of diagnostic group (SAD only or ASD+SAD), with the majority of participants displaying scores at or above clinical cut-offs. These findings indicate that performance on commonly used self-report measures of social anxiety does not differ between autistic adults with co- 
occurring SAD, relative to adults with SAD only. Taken in conjunction with the sound psychometric properties of these instruments in a large ASD cohort, our findings support the use of these instruments with autistic adults in clinical services.

The current study is not without its limitations, and many of these highlight potential directions for future research. Given the cross-sectional design of the study, we were unable to assess test-retest reliability. Additionally, our sample size, while considerably larger than prior studies in the field, prohibited us from assessing the factorial validity of these instruments in an autistic population. Thus, we are unable to ascertain whether these instruments display similar factor structures in an autistic population to those seen in SAD and typically developing populations. Future research would benefit from assessing the factorial validity of these instruments in autistic adults to further determine their utility and appropriateness in this population. Moreover, given that we did not include autistic participants with an IQ below 70, we acknowledge that our results can only be attributed to autistic individuals without an intellectual disability. We also acknowledge that not all autistic participants completed a diagnostic assessment for SAD; thus, we cannot ascertain how many autistic individuals in our cohort did not meet diagnostic criteria for SAD. However, based on the high prevalence of SAD in ASD, in conjunction with the observation that the majority of participants in our autistic cohort scored in the clinical range on the instruments reported, we would assume the proportion of autistic participants without comorbid SAD would be relatively small. Similarly, we did not complete the ADOS-2 on those individuals who were classified with a SAD diagnosis. Clinical screening at referral was used to determine whether individuals would need an ADOS-2 assessment to clarify an ASD diagnosis. We cannot rule out, however, that a small number of people in the SAD group may have met criteria for ASD. Finally, we cannot determine from the present results how sensitive these instruments are to change following treatment, although some previous 
research appears to show they may detect clinically significant changes (Spain, Blainey, \& Vaillancourt, 2017).

From a clinical perspective, these findings have important implications. Although many autistic adults experience considerable social anxiety, clinicians frequently report concerns about the lack of appropriate anxiety measures available for this group (Ainsworth et al., 2020). Based on our present findings, the LSAS-SR, SIAS, SPS and BFNE appear to be appropriate for use in autistic adults without intellectual disability, despite the difficulties that may be experienced by these individuals when reporting on their own emotions and affective states. As such, our findings support the use of these instruments as clinical tools to assess social anxiety symptoms and assist in the identification, diagnosis and treatment of SAD in autistic adults.

In conclusion, this study provides the first evidence that the LSAS-SR, SIAS, SPS and BFNE display adequate psychometric properties in an autistic cohort, and that autistic adults also diagnosed with SAD show similar summary scores and cut-off values on these instruments, compared to adults diagnosed with only SAD. Further research is now required to establish the factorial validity of these instruments in an autistic population. These results contribute to our understanding of the measurement of social anxiety symptoms in ASD and suggest opportunities for the implementation of these instruments in clinical settings servicing autistic adults. 


\section{Acknowledgements}

We thank the staff and research students who contributed to the broader dataset.

\section{Declaration of Conflicting Interest}

The Authors declare that there is no conflict of interest.

\section{Funding Acknowledgement}

The authors disclosed receipt of the following financial support for the research of this article: This work was supported by an Australian Research Council Linkage Grant [LP110200562]; and a National Health and Medical Research Council career development fellowship [GNT1056587] to AJG. 


\section{References}

Ainsworth, K., Robertson, A. E., Welsh, H., Day, M., Watt, J., Barry, F., . . Melville, C. (2020). Anxiety in adults with autism: Perspectives from practitioners. Research in Autism Spectrum Disorders, 69, 101457.

doi:https://doi.org/10.1016/j.rasd.2019.101457

Baker, S. L., Heinrichs, N., Kim, H.-J., \& Hofmann, S. G. (2002). The Liebowitz social anxiety scale as a self-report instrument: a preliminary psychometric analysis. Behaviour Research and Therapy, 40(6), 701-715. doi:https://doi.org/10.1016/S00057967(01)00060-2

Bejerot, S., Eriksson, J. M., \& Mörtberg, E. (2014). Social anxiety in adult autism spectrum disorder. Psychiatry Res, 220(1-2), 705-707. doi:10.1016/j.psychres.2014.08.030

Brown, T. A., \& Barlow, D. H. (2014). Anxiety and Related Disorders Interview Schedule for DSM-5 (ADIS-5). UK: Oxford University Press, Oxford.

Brown, T. A., DiNardo, P. A., \& Barlow, D. H. (1994). Anxiety Disorders Interview Schedule for DSM-IV. Oxford, UK.: Oxford University Press.

Carleton, R. N., Collimore, K. C., McCabe, R. E., \& Antony, M. M. (2011). Addressing revisions to the Brief Fear of Negative Evaluation scale: Measuring fear of negative evaluation across anxiety and mood disorders. Journal of Anxiety Disorders, 25(6), 822-828. doi:https://doi.org/10.1016/j.janxdis.2011.04.002

Cohen, J. (1988). Statistical Power Analysis for the Behavioral Sciences (2nd ed.). Hillsdale, NJ: Lawrence Erlbaum.

Crome, E., Grove, R., Baillie, A. J., Sunderland, M., Teesson, M., \& Slade, T. (2015). DSMIV and DSM-5 social anxiety disorder in the Australian community. Aust $N Z J$ Psychiatry, 49(3), 227-235. doi:10.1177/0004867414546699 
Gomez, R., \& Watson, S. D. (2017). Confirmatory Factor Analysis of the Combined Social Phobia Scale and Social Interaction Anxiety Scale: Support for a Bifactor Model. Front Psychol, 8, 70. doi:10.3389/fpsyg.2017.00070

Heimberg, R. G., Horner, K. J., Juster, H. R., Safren, S. A., Brown, E. J., Schneier, F. R., \& Liebowitz, M. R. (1999). Psychometric properties of the Liebowitz Social Anxiety Scale. Psychol Med, 29(1), 199-212. doi:10.1017/s0033291798007879

Hollocks, M. J., Lerh, J. W., Magiati, I., Meiser-Stedman, R., \& Brugha, T. S. (2019). Anxiety and depression in adults with autism spectrum disorder: a systematic review and meta-analysis. Psychological Medicine, 49(4), 559-572. doi:http://dx.doi.org/10.1017/S0033291718002283

Howlin, P., Goode, S., Hutton, J., \& Rutter, M. (2004). Adult outcome for children with autism. Journal of Child Psychology and Psychiatry, 45(2), 212-229. doi:10.1111/j.1469-7610.2004.00215.x

Howlin, P., \& Moss, P. (2012). Adults with autism spectrum disorders. Can J Psychiatry, 57(5), 275-283. doi:10.1177/070674371205700502

Howlin, P., Moss, P., Savage, S., \& Rutter, M. (2013). Social outcomes in mid- to later adulthood among individuals diagnosed with autism and average nonverbal IQ as children. J Am Acad Child Adolesc Psychiatry, 52(6), 572-581. doi:10.1016/j.jaac.2013.02.017

Leary, M. R. (1983). A Brief Version of the Fear of Negative Evaluation Scale. Personality and Social Psychology Bulletin, 9(3), 371-375. doi:https://doi.org/10.1177/0146167283093007

Liebowitz, M. R. (1987). Social phobia (pp. 141-173): S Karger AG. 
Lord, C., Luyster, R., Gotham, K., \& Guthrie, W. (2012). Autism Diagnostic Observation Schedule, Second Edition (ADOS-2) (2nd ed.). Torrance, CA: Western Psychological Services.

Lugnegard, T., Hallerback, M. U., \& Gillberg, C. (2011). Psychiatric comorbidity in young adults with a clinical diagnosis of Asperger syndrome. Res Dev Disabil, 32(5), 19101917. doi:10.1016/j.ridd.2011.03.025

Maddox, B. B., \& White, S. W. (2015). Comorbid Social Anxiety Disorder in Adults with Autism Spectrum Disorder. J Autism Dev Disord, 45(12), 3949-3960. doi:10.1007/s10803-015-2531-5

Mattick, R. P., \& Clarke, J. C. (1998). Development and validation of measures of social phobia scrutiny fear and social interaction anxiety. Behaviour Research and Therapy, 36, 455-470. doi:10.1016/s0005-7967(97)10031-6

Mazzone, L., Ruta, L., \& Reale, L. (2012). Psychiatric comorbidities in asperger syndrome and high functioning autism: diagnostic challenges. Annals of General Psychiatry, 11(16). doi:doi:10.1186/1744-859X-11-16

Mennin, D. S., Fresco, D. M., Heimberg, R. G., Schneier, F. R., Davies, S. O., \& Liebowitz, M. R. (2002). Screening for social anxiety disorder in the clinical setting: using the Liebowitz Social Anxiety Scale. Journal of Anxiety Disorders, 16(6), 661-673. doi:https://doi.org/10.1016/S0887-6185(02)00134-2

NICE. (2013). Social Anxiety Disorder: Recogntion, Assessment and Treatment. .

Nunnally, J. C. (1967). Psychometric Theory. New York: McGraw-Hill.

Park, S. H., Demetriou, E. A., Pepper, K. L., Song, Y. J. C., Thomas, E. E., Hickie, I. B., . . Guastella, A. J. (2019). Validation of the 36-item and 12-item self-report World Health Organization Disability Assessment Schedule II (WHODAS-II) in individuals with autism spectrum disorder. Autism Res, 12(7), 1101-1111. doi:10.1002/aur.2115 
Park, S. H., Song, Y. C., Demetriou, E. A., Pepper, K. L., Thomas, E. E., Hickie, I. B., \& Guastella, A. J. (under review). Validation of the 21-item Depression, Anxiety, and Stress Scales (DASS-21) in individuals with Autism Spectrum Disorder.

Peters, L. (2000). Discriminant validity of the Social Phobia and Anxiety Inventory (SPAI), the Social Phobia Scale (SPS) and the Social Interaction Anxiety Scale (SIAS). Behaviour Research and Therapy, 38, 943-950. doi:10.1016/s0005-7967(99)00131-x

Rodebaugh, T. L., Woods, C. M., \& Heimberg, R. G. (2007). The reverse of social anxiety is not always the opposite: the reverse-scored items of the social interaction anxiety scale do not belong. Behav Ther, 38(2), 192-206. doi:10.1016/j.beth.2006.08.001

Shattuck, P. T., Narendorf, S. C., Cooper, B., Sterzing, P. R., Wagner, M., \& Taylor, J. L. (2012). Postsecondary education and employment among youth with an autism spectrum disorder. Pediatrics, 129(6), 1042-1049.

Shrive, F. M., Stuart, H., Quan, H., \& Ghali, W. A. (2006). Dealing with missing data in a multi-question depression scale: a comparison of imputation methods. BMC Med Res Methodol, 6, 57. doi:10.1186/1471-2288-6-57

Spain, D., Blainey, S. H., \& Vaillancourt, K. (2017). Group cognitive behaviour therapy (CBT) for social interaction anxiety in adults with autism spectrum disorders (ASD). Research in Autism Spectrum Disorders, 41-42, 20-30. doi:10.1016/j.rasd.2017.07.005

Spain, D., Happé, F., Johnston, P., Campbell, M., Sin, J., Daly, E., . . Murphy, D. G. (2016). Social anxiety in adult males with autism spectrum disorders. Research in Autism Spectrum Disorders, 32, 13-23. doi:10.1016/j.rasd.2016.08.002

Spain, D., Sin, J., Linder, K. B., McMahon, J., \& Happé, F. (2018). Social anxiety in autism spectrum disorder: A systematic review. Research in Autism Spectrum Disorders, 52, 51-68. doi:10.1016/j.rasd.2018.04.007 
Swinscow, T. (1996). Statistics at Square One (9th ed.). London: BMJ Publishing Group [Revised by M J Campbell].

Terwee, C. B., D., B. S., de Boer, M. R., van der Windt, D. A., Knol, D. L., Dekker, J., . . de Vet, H. C. (2007). Quality criteria were proposed for measurement properties of health status questionnaires. J Clin Epidemiol, 60(1), 34-42. doi:10.1016/j.jclinepi.2006.03.012

Vasa, R. A., \& Mazurek, M. O. (2015). An update on anxiety in youth with autism spectrum disorders. Curr Opin Psychiatry, 28(2), 83-90. doi:10.1097/YCO.0000000000000133

Volkmar, F. R., Lord, C., Bailey, A., Schultz, R. T., \& Klin, A. (2004). Autism and pervasive developmental disorders. Journal of Child Psychology and Psychiatry, 45(1), 135170. doi:10.1046/j.0021-9630.2003.00317.x

Wechsler, D. (2001). Wechsler Test of Adult Reading: WTAR. San Antonio, TX: The Psychological Corporation.

Weeks, J. W., Heimberg, R. G., Fresco, D. M., Hart, T. A., Turk, C. L., Schneier, F. R., \& Liebowitz, M. R. (2005). Empirical validation and psychometric evaluation of the Brief Fear of Negative Evaluation Scale in patients with social anxiety disorder. Psychol Assess, 17(2), 179-190. doi:10.1037/1040-3590.17.2.179 


\section{Supplementary Materials}

\section{Supplementary Table 1}

Summary of missing data for groups. Figures represent number of participants who were missing values for each instrument and had data imputed using the individual mean of the remaining items.

\begin{tabular}{lcc}
\hline Instrument & $\begin{array}{c}\text { Social Anxiety Disorder } \\
(\mathrm{N}=316)\end{array}$ & $\begin{array}{c}\text { Autism Spectrum Disorder } \\
(\mathrm{N}=102)\end{array}$ \\
\hline LSAS-SR Total & 1 & 4 \\
\hline LSAS-SR Fear & 1 & 2 \\
\hline LSAS-SR Avoidance & 1 & 4 \\
\hline SIAS & 1 & 11 \\
\hline SPS & 1 & 7 \\
\hline BFNE & 0 & 7 \\
\hline BFNE-S & 0 & 7
\end{tabular}

Abbreviations: LSAS-SR, Liebowitz Social Anxiety Scale, self-report; SIAS, Social Interaction Anxiety Scale; SPS, Social Phobia Scale; BFNE, Brief Fear of Negative Evaluation Scale 


\section{Supplementary Table 2}

Correlations between instrument summary scores within the SAD group $(N=316)$

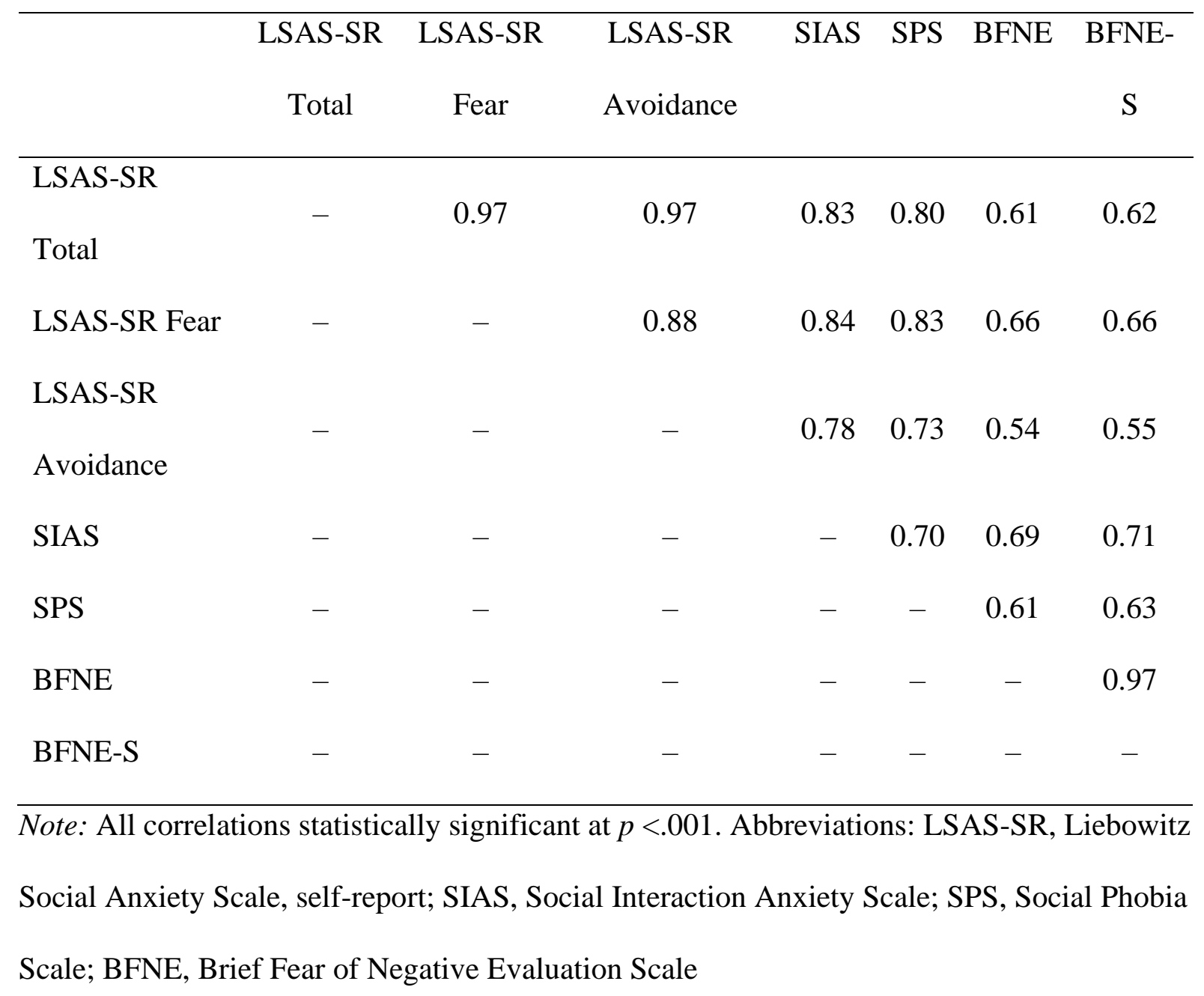




\section{Supplementary Table 3}

Correlations between instrument summary scores within the ASD group $(N=102)$

\begin{tabular}{|c|c|c|c|c|c|c|c|}
\hline & $\begin{array}{c}\text { LSAS-SR } \\
\text { Total }\end{array}$ & $\begin{array}{c}\text { LSAS-SR } \\
\text { Fear }\end{array}$ & $\begin{array}{l}\text { LSAS-SR } \\
\text { Avoidance }\end{array}$ & SIAS & SPS & BFNE & $\begin{array}{c}\text { BFNE- } \\
\text { S }\end{array}$ \\
\hline $\begin{array}{l}\text { LSAS-SR } \\
\text { Total }\end{array}$ & - & 0.96 & 0.96 & 0.81 & 0.81 & 0.64 & 0.68 \\
\hline LSAS-SR Fear & - & - & 0.84 & 0.80 & 0.85 & 0.70 & 0.75 \\
\hline $\begin{array}{l}\text { LSAS-SR } \\
\text { Avoidance }\end{array}$ & - & - & - & 0.74 & 0.70 & 0.52 & 0.55 \\
\hline SIAS & - & - & - & - & 0.71 & 0.67 & 0.69 \\
\hline SPS & - & - & - & - & - & 0.62 & 0.70 \\
\hline BFNE & - & - & - & - & - & - & 0.95 \\
\hline BFNE-S & - & - & - & - & - & - & - \\
\hline
\end{tabular}

Note: All correlations statistically significant at $p<.001$. Abbreviations: LSAS-SR, Liebowitz Social Anxiety Scale, self-report; SIAS, Social Interaction Anxiety Scale; SPS, Social Phobia Scale; BFNE, Brief Fear of Negative Evaluation Scale 


\section{Supplementary Table 4}

Fishers r-to-z transformations for correlation coefficients between SAD and ASD groups

\begin{tabular}{|c|c|c|}
\hline Pearson Correlation & $z$-score & $p$-value \\
\hline LSAS-Fear - LSAS-Avoidance & 1.31 & .190 \\
\hline LSAS-Fear - LSAS-Total & 0.78 & .435 \\
\hline LSAS-Fear - SIAS & 0.85 & .395 \\
\hline LSAS-Fear - SPS & -0.67 & .503 \\
\hline LSAS-Fear - BFNE & -0.67 & .503 \\
\hline LSAS-Fear - BFNE-S & -1.48 & .139 \\
\hline LSAS-Avoidance - LSAS-Total & 1.79 & .074 \\
\hline LSAS-Avoidance - SIAS & 0.73 & .465 \\
\hline LSAS-Avoidance - SPS & 0.27 & .549 \\
\hline LSAS-Avoidance - BFNE & 0.23 & .818 \\
\hline LSAS-Avoidance - BFNE-S & -0.07 & .944 \\
\hline LSAS-Total - SIAS & 0.68 & .497 \\
\hline LSAS-Total - SPS & -0.15 & .881 \\
\hline LSAS-Total - BFNE & -0.33 & .741 \\
\hline LSAS-Total - BFNE-S & -0.89 & .374 \\
\hline SIAS - SPS & -0.05 & .960 \\
\hline SIAS - BFNE & 0.32 & .749 \\
\hline SIAS - BFNE-S & 0.19 & .849 \\
\hline SPS - BFNE & -0.11 & .912 \\
\hline SPS - BFNE-S & -1.14 & .254 \\
\hline BFNE - BFNE-S & 1.43 & .153 \\
\hline
\end{tabular}


Note: Negative $z$-score indicates that the correlation coefficient in the SAD group was weaker than that in the ASD group. Abbreviations: LSAS-SR, Liebowitz Social Anxiety Scale, selfreport; SIAS, Social Interaction Anxiety Scale; SPS, Social Phobia Scale; BFNE, Brief Fear of Negative Evaluation Scale 